\title{
Remarks on Simple Relativity and the Relative Velocity of Light.
}

\author{
By Sir Oliver Lodge, F.R.S.
}

I.

I $\mathrm{N}$ continuation of my article in the Relativity Number of Nature (vol. cvi., p. 795, February 17,1921 ), I propose to discuss more fully, and to express as clearly and simply as possible, some of the points on which philosophic disciples or expounders of Einstein have written, so as perhaps to remove a certain amount of misapprehension, and incidentally to set my own views before other physicists, in order that they may be controverted where necessary. On some other points of more general interest $I$ have written in the Fortnightly Review for next September, especially on the foundation which had been laid by Einstein's predecessors before the philosophic doctrine of relativity was made definite and erected into a comprehensive physical theory.

The Fundamental Relativity Hypothesis.

Einstein's first fundamental assumption is that direct observation of our absolute motion through space is not only unachieved, but also in the nature of things impossible; wherefore it can be held that such motion has no intelligible meaning. Those who admit an æther prefer not to shut the door on inquiry, but meanwhile express their provisional agreement by saying that its various functions and properties are so uniform, so universal, and so interrelated, that observation of any suspected effect of motion through the æther is liable to be frustrated or negatived by some-so to say-inevitable opposite effect; and that the compensation, at any rate over a wide range, is complete.

Einstein's second fundamental assumption is that the one absolute quantity which can be observed, namely, the velocity of light-if it be a velocity-is unique and so fundamental that every observer must necessarily measure the same result if he make his measurements correctly, no matter what his own motion may be; which, after all, is only another way of saying that his own motion through the æther is pragmatically a meaningless expression.

It is not claimed that these assumptions, which are certainly consistent with the Larmor-Lorentz transformation equations-at least, when they include the factor $\beta$, expressive of the FitzGeraldLorentz contraction-are really established by them. That would be reasoning in a circle. Nor do the equations necessarily substantiate any metaphysical assertions about time or space or æther; but they do lead to algebraic and legitimate deductions.

\section{The Time and Space Transformation.}

The importance of those transformations-correlating the states of the same material system travelling at different speeds-can scarcely be exaggerated. They have been arrived at in many ways, usually by aid of ideal and hypothetical and apparently impossible experiments, sometimes by considering that an event does not effectively happen until we have seen it happen, thus entailing relative delay; and they have been variously interpreted. The original gist of the equations was that a moving observer must not only take his distances as variable; he must consider his times variable too. He must have a local and fictitious time peculiar to himself, if he is to ignore his own motion and treat his direct measurements as conclusive.

Einstein's step was equivalent to dispensing with any overt fiction about this subjective or local measure of time, to claiming that it was as real as any other, though peculiar to each observer, and to seeing what emerged.

Now if we agree to waive any question of experimental practicability, and proceed in an ideal fashion, it is easy enough to obtain notions about the required transformation; and as I have not seen the equations obtained so directly or naively, I proceed to deduce them thus :-

A stationary observer, supposed able to time the passage of light from a source at a distance $x$, may be expected to get the result

$$
\frac{x}{t}=c .
$$

If he be moving towards the source with speed $u$, he will be relieving the light of some of the journey by doing that bit himself. The light need now only travel a smaller distance $x^{\prime}$ to meet him, and the observer will have travelled the remainder, namely, $x-x^{\prime}$. So if the time taken on the jointly performed journey be $t^{\prime}$, and if he finds it possible to measure the distance $x^{\prime}$ at the instant the light reaches him, which is evidently the right moment, he will get

$$
\begin{aligned}
& x^{\prime} \\
& t^{\prime}
\end{aligned}=c \quad \text { as the speed of the light, }
$$

and

$$
\frac{x-x^{\prime}}{t^{\prime}}=u \quad \text { as his own speed. }
$$

Given these three equations, we get by mechanical algebra without further reasoning

$$
t=t^{\prime}+\frac{u x^{\prime}}{c^{2}}
$$

as well as the more obvious

$$
x=x^{\prime}+u t^{\prime}
$$

without mentioning relativity at all.

If all these measurements could be really made, we should have

$$
c+u=\frac{x}{t^{\prime}},
$$

and $u$ could be determined in terms of $c$. But the measurements are impracticable as they stand, for how is an observer to know the instant at which a particular portion of light left the source? In other words, how is he to time an event on the source when he is dependent on the light itself for information of its occurrence? He might have the event telegraphed, but that information also is transmitted by the æether at the same pace. So the foiled inquirer will naturally try to get some additional data by reversing his motion and starting back again from 
his present position at distance $x^{\prime}$, so as to be moving away from the light instead of towards it. The light will now have to catch him up; and he may think, at first, that the ray which left the source at the instant he began his return journey will take the original time $t$ to reach him, since it now has to travel the full distance $x$. But he will have to travel a little further than the original position, and take a little longer time, before he is overtaken; and he cannot write the reciprocal equations

and

$$
t^{\prime}=t-\frac{u x}{c^{2}}
$$

$$
x^{\prime}=x-u t,
$$

because they are inconsistent with the previous ones.

To make the two pairs of equations agree (as relativity demands), either $x$ must equal $x^{\prime}$, which frustrates the whole experiment, or a common factor must be introduced, say $\beta$, such that

and

$$
t^{\prime}=\beta\left(t-\frac{u x}{c^{2}}\right), \text { or } \beta t\left(1-\frac{u}{c}\right)
$$

$$
t=\beta\left(t^{\prime}+\frac{u x^{\prime}}{c^{2}}\right), \text { or } \beta t^{\prime}\left(\mathrm{I}+\frac{u}{c}\right) \text {. }
$$

This will render them harmonious, and a suitable value (the only right value) of $\beta$ is easily reckonedagain mechanically, without further hypothesisnamely,

$$
\beta^{2}\left(\mathrm{I}-\frac{u^{2}}{c^{2}}\right)=\mathrm{I} .
$$

If that is satisfied, the reversal of the journey will not give any different result; there is perfect reciprocity. You cannot by an experiment of reversing your motion with regard to light, or reflecting back the light with regard to the observer, discriminate between $c-u$ and $c+u$; nor can you discriminate either from $c$.

Now this $\beta$ factor is the FitzGerald-Lorentz contraction; the experiment thus neutralised is the Michelson-Morley experiment; and the direct supposition that an observer must find $c-u$, and $c$, and $c+u$ all the same, or at least indistinguishable by observation, and that there is nothing more to be said, is the point of view of Einstein.

These names must suffice to suggest a flood of ideas to those who have read about the subject.

To sum up compactly:-

Assume that you cannot help measuring the same speed of light whether you be moving or stationary, so that $x / t$ and $x^{\prime} / t^{\prime}$ both equal $c$ (the accented letters referring to the measurements made when you were moving with speed $u$ to meet the light), then allow that $x / t^{\prime}$ is not equal to $c+u$, as you would expect, nor $x^{\prime} / t$ equal to $c-u$ (for in that case $x x^{\prime} / t t^{\prime}$ would equal $c^{2}-u^{2}$ instead of $c^{2}$ ), but that, instead,

$$
\frac{x}{t^{\prime}}=\beta(c+u) \text { and } \frac{x^{\prime}}{t}=\beta(c-u),
$$

which, together, require that

$$
\beta^{2}=\frac{c^{2}}{c^{2}-u^{2}}
$$

then all the rest follows.

\section{The Contraction.}

A customary and older interpretation of the introduction of the factor $\boldsymbol{\beta}$-to complete and make accurate what then became the LarmorLorentz transformation-is that the measuring rod with which you are hypothetically supposed to measure $x$ or $x !$ shrinks to $\mathrm{I} / \boldsymbol{\beta}$ of its normal NO. 2701 , VOL. IO7] length if the experimenter is moving either to or fro with speed $u$, so that all distances in the direction of motion measure out a little bigger than they otherwise would; more steps of the yard measure having to be taken. Note that space or æther does not shrink, but only the matter in space. The distance $x$ has not changed, but only the instrument with which you hypothetically measure it. That having shrunk, the fixed distance measures out longer. The same thing happens with the instrument whereby you are supposed to measure time. Both distance and time of journey are abbreviated by approach, but, to measurement, not so much as an unchanged measurer would give. They are both lengthened by recession, and the measurements give rather more increment than might have been expected.

The ratio between measurements made during uniform approach, and the same made during relative rest, is

$$
\frac{x^{\prime}}{x}=\frac{t^{\prime}}{t}=\beta\left(\mathrm{I}-\frac{u}{c}\right)=\sqrt{\left(\frac{c-u}{c+u}\right)} .
$$

This line, with the definition $c=x / t$, is the briefest possible summary of the transformation equations.

A short and easy way of getting, or at least of recording, the essence of the transformation is to allow for the contraction of the hypothetical measuring rod by multiplying any distance across space supposed to be measured by a flying observer-flying towards or away from a distant event which really occurred at the instant he started to fly-by an undefined numerical coefficient $\beta$, and omitting this factor from any distance which he could have measured at rest before starting.

Thus let an event occur at the origin, and let an observer at $x$ and $t$ immediately begin travelling towards it, so as to meet the light at a place which appears to him to be $x^{\prime}$ and $t^{\prime}$, the combined velocity over the original distance being $c+u$, he can correct his $x^{\prime}$ measurement, which has been traversed by the light alone, and write

$$
\frac{x}{c+u}=\frac{\beta x^{\prime}}{c}
$$

while if he started from the leisurely measured $x^{\prime}$ and $t^{\prime}$ position, directly the event occurred at the origin, and receded so that the light overtook him at what appears to him to be a place $x$ and $t$, coming with the relative velocity $c-u$, he can correct his $x$ measurement for the whole distance traversed by the light, and write

$$
\frac{x^{\prime}}{c-u}=\frac{\beta x}{c}
$$

saying, if he likes, that it is just the same as if he had stood still and the light had come to him with diminished speed. (Or he might time his own journey as $\frac{\beta x-x^{\prime}}{u}$, and equate that to $\left.\frac{\beta x}{c}\right)$. Combining these equations with the definition

$$
\frac{x^{\prime}}{t^{\prime}}=\frac{x}{t}=c,
$$

and not troubling about the $y$ and $z$ co-ordinates, which remain unchanged and need no attention, we get the Lorentz transformation complete (and incidentally we see that the usual differential invariant $d s^{2}=d x^{2}+d y^{2}+d z^{2}-c^{2} d t^{2}$ is always zero for light). 


\section{Once the transformations}

$\left\{\begin{array}{l}x^{\prime}=\beta(x-u t) \\ t^{\prime}=\beta\left(t-\frac{u x}{c^{2}}\right)\end{array}\right.$ with their correlative $\left\{\begin{array}{l}x=\beta\left(x^{\prime}+u t^{\prime}\right) \\ t=\beta\left(t^{\prime}+\frac{u x^{\prime}}{c^{2}}\right)\end{array}\right.$

are introduced, the coefficient $\beta$ is self-defined as $\beta^{2}\left(c^{2}-u^{2}\right)=c^{2}$, and results flow in thick and fast. Thus if we seek to superpose a velocity $d x^{\prime} / d t^{\prime}$, or $v$, on the speed $u$, and reckon the result as $d x / d t$, or $w$, working mechanically on the above two equations, we do not find, as we might expect, $w=u+v$, but

$$
v=\frac{u+v}{\mathrm{I}+\frac{u v}{c^{2}}}
$$

This appears to have nothing to do with the $\boldsymbol{\beta}$ factor, but to depend only on the second term in the expression for $t$. We must remember, however, that without the $\beta$ factor we could not write the reciprocal equations, which permit simple reversal of sign in $v$ if it is opposed to $u$. The consequences of this law of composition of velocities are astonishing, and include among them the uniqueness and maximal character of the velocity $c$.

\section{Confirmation.}

The Einstein assumptions have never been directly ascertained by experiment. They are not the result of experiment at all; they are a reasoned type of hypothesis, and any provisional confirmation must be derived from the legitimacy of the conclusions which, from them and their extensions, the far-reaching genius of Einstein has shown to be attainable. Briefly we may cite the general type of confirmations, including those of his completer theory, thus :-

The velocity of light inside transparent matter, being less than its value in free space, is affected by its motion in the way Fresnel predicted and Fizeau confirmed. The equations give this result without the need of Fresnel's theory.

Even outside great masses of matter the velocity is now discovered to be slightly affected (still diminished, never increased) in a second-order way that Einstein predicted and astronomers confirmed. Starlight is deflected by this gravitational refractivity.

Not only so. The neighbourhood of a very large mass of matter introduces secondary higherorder effects into the æther in such a way as to affect not its luminiferous properties only, but its gravitational structure too; and the consequence is that the orbit of a planet sufficiently near the sun behaves, not exactly in accordance with the laws of particle dynamics in empty space, but with a slight modification, depending on the squares of small quantities, such as the general principle of relativity enabled Einstein to calculate. And, as everyone knows, an outstanding discrepancythough one detected only through the extreme refinement of astronomy-was thus triumphantly removed from the planet Mercury, the only planet near enough to the sun to be sensibly affected.

NO. 2701, VOL. IO7]
Thus, then, the general mathematical trend of ideas on which the principle was founded may be claimed as confirmed in this ex post facto manner; but many varieties of expression, and attempts to interpret the principle philosophically, are far from establishment still.

\section{Alternative Modes of Statement.}

If we take up an agnostic position, we cannot say-and Prof. Einstein seems to agree-that, as a deduction from experiment, any philosophic or metaphysical position is really proven. What we can definitely say is that certain statements are consistent with all the experiments hitherto made, but we cannot say that every other mode of statement is riled out. In nearly every case-probably in every case- the result of experiment can be expressed otherwise. Thus, for instance, my experiment with the rotating discs (Phil. Trans., vol. clxxxiv., I893) showed that their motion neither added nor subtracted anything, nor affected the velocity of light in their immediate neighbourhood, although their circumference was travelling at a speed almost sufficient to tear the steel asunder, and although an exceedingly minute alteration in the speed of light could have been observed; but this negative result can be expounded, and indeed was expounded, by saying that the æther - the vehicle of light-is not carried forward or perturbed at all by the adjacent moving matter. And that is part of an entirely rational wther theory of the atomic structure of matter.

The famous Michelson-Morley experiment, again, wherein no result is found, although the apparatus must be immersed in a relative æther stream, can be, and was, explained by saying that. every solid body suffers a FitzGerald-Lorentz deformation due to its motion relative to that stream.

Again, the most important Fizeau experiment, one which did yield a positive result, because here light was travelling through and inside transparent moving matter, and so was accelerated and" retarded by a measurable amount-not, indeed, beyond the velocity $c$, but beyond the velocity $c / n$, where $n$ is the index of refraction--this result was explained, and by Fresnel anticipated, by assuming (crudely) that a given proportion of the æther clung to moving matter and was transported with it, or (less crudely) that the presence of matter so modified or loaded the æther as not only to retard the light considerably in any case, but to retard it differently when in motion than when at rest. Electrically, this comes out with complete clarity, because the loading property-the mattercaused modification of the ather constants $\mu$ and $\mathrm{K}$-really does belong to the matter, and travels. with it.

So in every instance which had been already explored an explanation was forthcoming, and had been accepted as sufficiently plausible and satisfactory; but it was a different explanation in each case. Not differing so as to be inconsistent - they were all consistent with a certain view of 
the æther, and were all in agreement with the electrical theory of matter-but still, when Einstein showed that the law of composition of velocities appropriate to his principle of relativity accounted for them all as an immediate corollary, without effort and without any assumption beyond what was embodied in that principle-this feature of directness naturally aroused the keen attention of physicists.

(Discussion of the relative velocity of light is deferred to next week.)

(To be continued.)

\section{Endowment of Scientific Research in the United States. ${ }^{1}$}

$I^{N}$ Nature for May 29, I9I9, an account was given of the organisation of the National Research Council of the United States of America. Supported during the war largely by the Government, but now entirely by private bodies and firms (it has lately received a grant of 5,000,000 dollars from the Carnegie Corporation), this body owes its existence to a trend of opinion by no means confined to the capitalist classes which maintain it. The American Federation of Labour explicitly and emphatically professed its belief in the fundamental importance and beneficent results of scientific research-more especially research in pure science-in a manifesto quoted in the Report for I9I8-i9 of our own Department of Scientific and Industrial Re. search. This unanimity on the part of employer and employed in their recognition of the importance for the development of American industries of the promotion of research gives additional weight to the imposing array of facts and figures assembled by the National Research Council in the bulletin under notice, which deals with funds, other than Federal and State funds, available in 1920 for this purpose.

In the preparation of the following summary it has been assumed that where the total endowment, but not the amount annually available, is given in the bulletin, 5 per cent. of this total was available. In some cases no information is given as to the amount of the fund-either capital or interest-and these were necessarily omitted in compiling the money totals. The columns A, F, $\mathrm{U}$ give the number and aggregate annual value in thousands of dollars of the funds provided by, or in connection with:- $\mathrm{A}$ : academies, associations, societies, and museums; $F$ : foundations, hospitals, and research institutes; $U$ : universities and colleges.

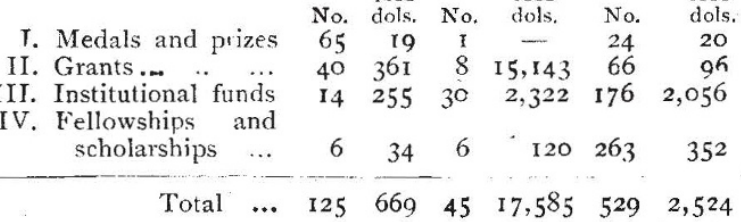

The most conspicuous figure in this table is the amount of the grants by foundations, etc., and this is almost entirely composed of appropriations (amounting to $15,000,000$ dollars) made by the Rockefeller Foundation, New York City, "partly

1 "Funds available in r920 in the United States of America for the Encouragement of Scientific Research." Bulletin No. 9 of the National Research Council, r7or Massachusett. Arenue, Washington, D.C., March, rو2I. I dollar.

NO. 2701, VOL. IO7] to agencies which it creates for carrying out specific programmes, and partly to other existing organisations to enable them to carry out specific programmes." Several other important annual appropriations are detailed below.

Amount
(roo dols.)

Rockefeller Institute for Medical Research I, 100

Carnegie Institution of Washington-for research in astronomy, 22I ; physics, 329 ; botany, 65 ; biology, 13 I ; nutrition, $5^{2}$; eugenics, 3I; embryology, $43 \ldots \ldots, \ldots$

Carnegie Institution, minor grants ... American Museum of Natural History-
for promotion of research, exploration, $\begin{array}{lllllll}\text { etc. } \quad \ldots & \ldots & \ldots & \ldots & \ldots & \ldots & 278\end{array}$

Harvard Fund for medical research $\quad \ldots \quad r \quad 363$

J. De Lamar Funds for study and teaching of dietetics and of the origin, etc., of

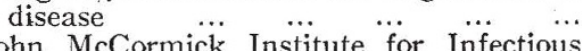

John McCormick Institute for Infectious
Diseases Research Fund National Research Council fellowships for research in physics and chemistry ...

Massachusetts Institute of Technology, general budget appropriation for re$\begin{array}{lllllll}\text { search } & \ldots & \ldots & \ldots & \ldots & \ldots & 100\end{array}$

Thus, of the aggregate amount of the sums specified in the bulletin-20,778,00o dollarsmore than 82 per cent. is attributable to Rockefeller and Carnegie benefactions, and more than 88 per cent. to these and the six other sources specified.

At the recent congress at Oxford of representatives of the universities of the British Empire much emphasis was laid on the funda: mental importance of scientific research and on the necessity for providing material aids and training for it. The figures given above constitute a striking commentary. on the following observations made by Prof. Joly at the congress: "Perhaps the most striking feature of American universities, as viewed by the British visitor, is the prevalence of research, and the lavish provision made for its prosecution. . . . There is research in everything. The American recognises to the full the value of the mental attitude induced by research, and this recognition is not confined to the university professor, from whom it may be expected, but extends, so far as I could gather, everywhere throughout the States." At some future date the National Research Council will perhaps take stock of the results of the application of these vast sums of money, and may possibly have a tale to tell of misdirected or unfruitful effort; but it can scarcely be doubted that the net results will affect substantially the welfare of mankind-perhaps so 